\title{
Commentary: A Human Pluripotent Stem Cell-Based Platform to Study SARS-CoV-2 Tropism and Model Virus Infection in Human Cells and Organoids
}

\author{
Amin Ardestani ${ }^{1,2}$ and Kathrin Maedler ${ }^{1 *}$ \\ ${ }^{1}$ Centre for Biomolecular Interactions Bremen, University of Bremen, Bremen, Germany, ${ }^{2}$ Department of Molecular \\ Medicine, School of Advanced Technologies in Medicine, Tehran University of Medical Sciences, Tehran, Iran
}

Keywords: COVID-19, SARS-CoV-2, diabetes, beta-cell, inflammation, cytokine storm

\section{OPEN ACCESS}

Edited by:

Gaetano Santulli,

Columbia University, United States

Reviewed by:

Kazuo Takayama,

Kyoto University, Japan

*Correspondence:

Kathrin Maedler

kmaedler@uni-bremen.de

Specialty section:

This article was submitted to

Clinical Diabetes,

a section of the journa

Frontiers in Endocrinology

Received: 19 August 2020 Accepted: 28 September 2020

Published: 14 October 2020

Citation:

Ardestani A and Maedler K (2020) Commentary: A Human Pluripotent Stem Cell-based Platform to Study

SARS-CoV-2 Tropism and Model

Virus Infection in Human

Cells and Organoids.

Front. Endocrinol. 11:585922. doi: 10.3389/fendo.2020.585922

\section{A Commentary on}

A Human Pluripotent Stem Cell-Based Platform to Study SARS-CoV-2 Tropism and Model Virus Infection in Human Cells and Organoids

By Yang L, Han Y, Nilsson-Payant BE, Gupta V, Wang P, Duan X, et al. (2020). Cell Stem Cell 27:125-36 e7. doi: 10.1016/j.stem.2020.06.015

\section{INTRODUCTION}

The COVID-19 pandemic caused by the SARS-CoV-2 coronavirus has become a major threat for our society at multiple levels. SARS-CoV-2 has spread around the world and as per today more than 30 Million people have been infected (World Health Organization). Although it had been suggested at the beginning of the outbreak that a herd immunity with controlled infections should be achieved, we now know of severe complications initiated by SARS-CoV-2, especially critical for metabolic diseases. While COVID-19 mortality is mainly caused by pulmonary failure, a deleterious bidirectional relationship between COVID-19 and diabetes has been observed in several recent studies (1) such that (i) diabetes and the metabolic syndrome are associated with an up to 50\% increased risk of fatal and severe COVID19 for patients with diabetes compared to those who do not have diabetes (2). Furthermore, uncontrolled elevated blood glucose levels are associated with highest COVID-19 mortality rates (3, 4). And (ii) COVID-19 induces severe metabolic complications of pre-existing diabetes such as ketoacidosis and even acute diabetes onset or type 1 diabetes (T1D) $(1,5-9)$.

\section{A Human Organoid Platform to Study SARS-CoV-2 Tropism}

Yang et al. now provide a unique platform of human cell and organoid models for the SARS-CoV-2 tropism to understand the whole picture of SARS-CoV-2's effects on various cells and tissues (10). From the tested human pluripotent stem cells (hPSC)-derived cells of all three definitive germ layers, namely, pancreatic $\alpha$-, $\beta$ and $\delta$-cells, liver organoids, endothelial cells, cardiomyocytes, macrophages, microglia, and cortical and dopaminergic neurons, they found that pancreatic glucagon-positive $\alpha$ - and insulinpositive $\beta$-cells, liver organoids, cardiomyocytes, and dopaminergic neurons are specifically permissive to 
SARS-CoV-2 virus infection (Figure 1A). The potential permissiveness of cells and organoids to SARS-CoV-2 viral entry achieved by a commonly used vesicular stomatitis virus (VSV)based SARS-CoV-2 pseudo-entry virus with an incorporated SARSCoV-2 spike protein was confirmed in hPSC-derived primary cells, namely, in human hepatocyte and cholangiocyte organoids and in pancreatic endocrine cells and in isolated primary human islets. Also, an in vivo model with hPSC-derived endocrine islet-like cells transplanted under the kidney capsule of humanized mice shows robust SARS-CoV-2 entry and efficiency of virus infection

A
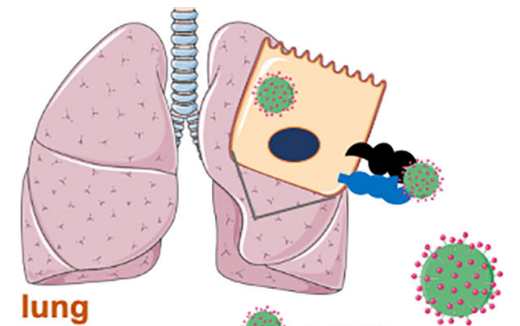

SARS-CoV-2

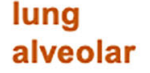
ACE2 alveola
cells infection

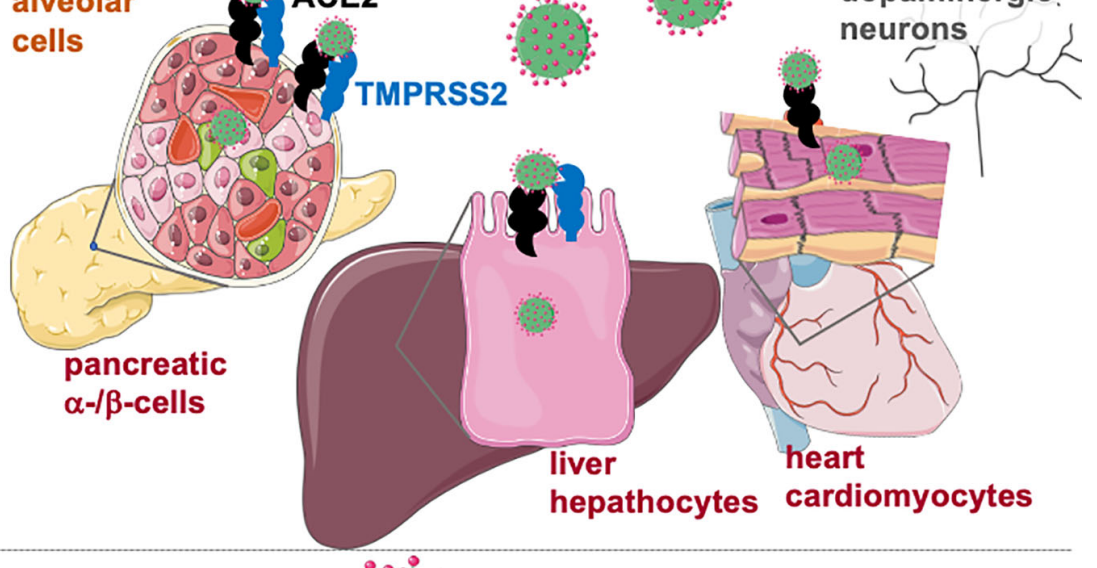

B

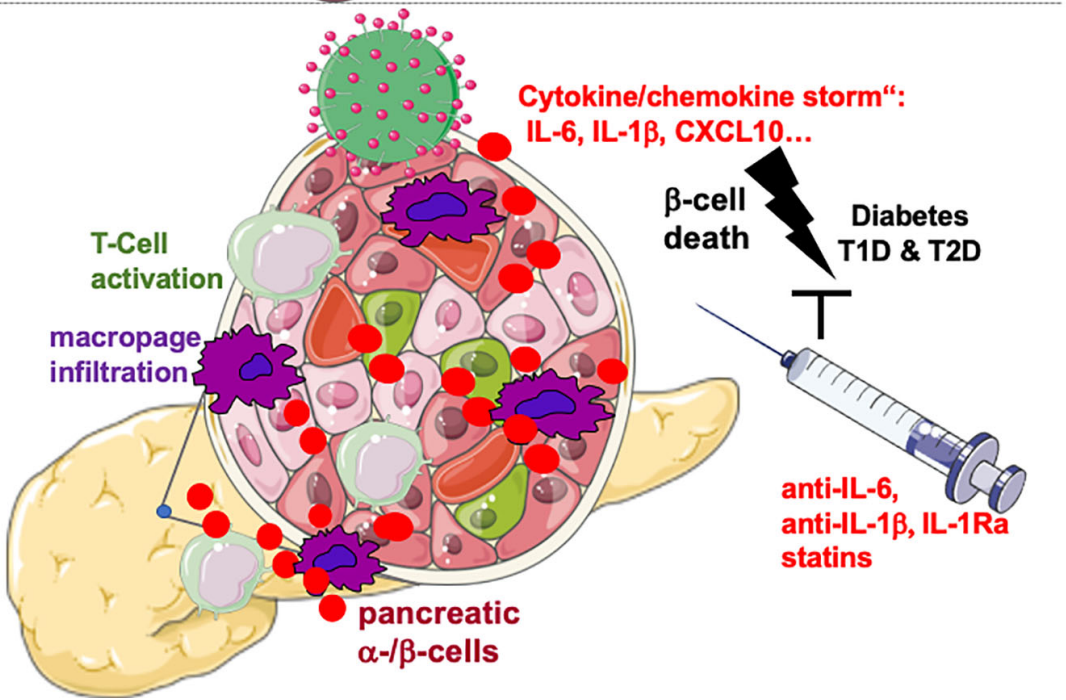

FIGURE 1 | Lung alveolar cells, pancreatic $\alpha$ - and $\beta$-cells, hepatocytes, cardiomyocytes, and dopaminergic neurons are specifically permissive to SARS-CoV-2 virus infection. (A) SARS-CoV-2 enters cells through the ACE2 receptor and the effector protease TMPRSS2, both expressed on lung alveolar cells and on many other cells in the body, although viral load does not essentially correlate with their expression. Organs affected by the metabolic syndrome are specifically permissive to SARS-CoV-2 infection which is a potential reason for the metabolic deterioration and the more severe disease, seen in COVID-19 patients with obesity and diabetes. (B) The "cytokine storm", i.e. the massive cytokine production by SARS-CoV-2 infected cells may lead to destruction of insulin producing $\beta$-cells in the pancreas, which are specifically vulnerable to inflammatory cell death cascades. Consequent activation of cytotoxic $\mathrm{CD}^{+}$and $\mathrm{CD} 8^{+} \mathrm{T}$-cells and migration of macrophages may initiate auto-immunity and acute onset of diabetes after COVID-19. Anti-inflammatory therapies, which have been shown to protect $\beta$-cells from the vicious cycle of cytokines need to be evaluated for an add-on anti-viral therapy for COVID-19 patients with diabetes or at risk for T2D or T1D. Created using smart servier medical art under https://creativecommons.org/licenses/by/3.0/ . 
specifically in $\alpha$ - and $\beta$-cells of the graft. In primary human islets, SARS-CoV-2 viral infection correlates with the expression of ACE2, the receptor for SARS-CoV-2, expressed in $\alpha$ - and $\beta$-cells based on single-cell RNA sequencing (sc-RNA) and immunostaining. The prominent $\beta$-cell ACE2 expression was confirmed by three other recent studies, together with its upregulation by inflammation (11) and type 2 diabetes (T2D) (12); a generally slightly higher ACE2 expression was shown in the pancreas in endocrine, acinar and ductal cells, as compared to lung cells (13). In contrast, detailed analyses of RNA-seq data sets revealed very low frequency and expression levels of ACE2 in islets and no differences between nondiabetic and T2D donors $(14,15)$. In line with the low ACE2 expression in islets, SARS-CoV-2 nucleocapsid protein was primarily expressed in ducts only seen in one out of three investigated autopsy pancreases from COVID-19 patients (14). To resolve the controversy, further detailed histology experiments using the ACE2 antibodies already evaluated in these studies in ACE2 depleted cells are required.

Nevertheless, cellular ACE2 expression itself is not an indicator of SARS-CoV-2 infection in general, as the authors found low or even no viral entry into endothelial cells, macrophages or cortical neurons despite ACE2 expression. Also, the SARS-CoV-2 effector protease, transmembrane serine protease 2 (TMPRSS2), which is currently investigated for antiviral therapy, does not correlate with cellular infection. While highly expressed in the pancreas, it is undetectable in the heart (https://www.proteinatlas.org/), despite viral entry into cardiomyocytes (10). Thus, based on all these recent studies, the role of cellular ACE2 expression in the pathophysiology of COVID19 remains unclear.

\section{COVID-19 and Diabetes: An Inflammatory Round Trip}

The mechanism for the mortal relationship of COVID-19 and diabetes has been suggested to be associated with the SARS-CoV-2 induced "cytokine storm" through the excessive interferon response to inhibit viral replication. However, if overly, it induces massive tissue damage. Transcriptomic analysis of SARS-CoV-2 virus infected hPSC-derived pancreatic endocrine cells as well as hepatocyte organoids confirmed this hypothesis with the striking upregulation of classical viral sensitive pathways, namely chemokine and cytokine response, apoptotic cell death genes and marked downregulation of key signaling transcripts associated with hormonal functions of endocrine cells, including calcium signaling pathways or key hepatocyte metabolic markers of the Cytochromes P450 superfamily of enzymes in the liver. Notably, cytokine and chemokine profiles linked to the viral response are consistent with those upregulated in lung autopsy samples from COVID-19 patients compared to control donors, including CXCL5, CXCL6, CXCL10, and IL-1 $\beta$ (10). However, all data are based on RNA analyses and it will be important to confirm cytokine protein expression and secretion in future studies.

\section{DISCUSSION}

The concept of virus associated diabetes is not new. Already back in 1926, Franklin Adams observed that severe T1D breaks out "immediately after such an infection", and this has been later confirmed in many large studies (16). There is a large body of evidence for enteroviral infection initiating the auto-immune response and subsequent $\beta$-cell destruction in genetically predisposed individuals, where a viral response is boosted through massive production of pro-inflammatory cytokines. Decorated with a high concentration of pattern recognition receptors, i.e., TLR3/4, RIG-I, MDA-5, and with IL-1R1, and especially vulnerable to inflammatory destruction, the $\beta$-cell undergoes apoptosis in response to inflammation, metabolic control derails and diabetes develops. In parallel, the virus initiated interferon response accelerates hyperexpression of surface HLA-I molecules and thus activation of auto-reactive T-cells and auto-immunity against $\beta$-cells (16). Also, $\beta$-cell failure in $\mathrm{T} 2 \mathrm{D}$ has been associated with chronic subclinical inflammation and elevated cytokines and chemokines, especially IL-1 $\beta$, IL-6, and CXCL10 levels in proximity to the islet are initiator for $\beta$-destruction, dysfunction, and metabolic deterioration (Figure 1B) $(16,17)$.

Furthermore, highly elevated serum IL-6, IL-8 and TNF- $\alpha$ in patients with COVID-19 are strong and independent predictors of COVID-19 severity and survival (18), which suggests the „cytokine storm“ indeed as crucial target for therapy. As neither effective antiviral drugs nor approved effective vaccination are currently available, major efforts are needed to treat the deleterious effects of SARS-CoV-2 infection, especially for patients at high risk to develop diabetes; T2D and T1D, obese patients with impaired insulin sensitivity or patients with a family history of auto-immunity. The powerful current stateof-the-art anti-inflammatory corticosteroid therapy (e.g., dexamethasone) needs to be carefully evaluated in patients with diabetes, because of their well-known secondary diabetogenic adverse effects at high doses. Rather, specific and promising anti-inflammatory compounds, such as anti-IL-6, anti-IL-1, and IL-1Ra, which showed robust reduction in markers of inflammation and improvement in glycemia in many clinical trials (17), or lipid lowering anti-inflammatory statins (19) should be considered for the symptomatic therapy for COVID-19 patients with diabetes (Figure 1B).

Since investigations have been performed in iPS-derived islet cells or human islets in vitro, immediate next steps would be to get access to more autopsy samples from COVID-19 patients, especially from the heart, liver, and pancreas (1) to analyze the virus permissiveness and infection efficiency in these organs and (2) to characterize virus infected cells in patients.

\section{AUTHOR CONTRIBUTIONS}

AA and KM wrote this article. All authors contributed to the article and approved the submitted version.

\section{FUNDING}

German Research Foundation (DFG) and JDRF. 


\section{REFERENCES}

1. Rubino F, Amiel SA, Zimmet P, Alberti G, Bornstein S, Eckel RH, et al. NewOnset Diabetes in Covid-19. N Engl J Med (2020) 383:789-90. doi: 10.1056/ NEJMc2018688

2. Bornstein SR, Rubino F, Khunti K, Mingrone G, Hopkins D, Birkenfeld AL, et al. Practical recommendations for the management of diabetes in patients with COVID-19. Lancet Diabetes Endocrinol (2020) 8(6):546-50. doi: 10.1016/S2213-8587(20)30223-0

3. Zhu L, She ZG, Cheng X, Qin JJ, Zhang XJ, Cai J, et al. Association of Blood Glucose Control and Outcomes in Patients with COVID-19 and Pre-existing Type 2 Diabetes. Cell Metab (2020) 31(6):1068-77 e3. doi: 10.1016/j.cmet.2020.04.021

4. Wang S, Ma P, Zhang S, Song S, Wang Z, Ma Y, et al. Fasting blood glucose at admission is an independent predictor for 28-day mortality in patients with COVID-19 without previous diagnosis of diabetes: a multi-centre retrospective study. Diabetologia (2020) 63:2102-11. doi: 10.1007/s00125-020-05209-1

5. Chee YJ, Ng SJH, Yeoh E. Diabetic ketoacidosis precipitated by Covid-19 in a patient with newly diagnosed diabetes mellitus. Diabetes Res Clin Pract (2020) 164:108166. doi: 10.1016/j.diabres.2020.108166

6. Yang JK, Lin SS, Ji XJ, Guo LM. Binding of SARS coronavirus to its receptor damages islets and causes acute diabetes. Acta Diabetol (2010) 47(3):193-9. doi: 10.1007/s00592-009-0109-4

7. Obukhov AG, Stevens BR, Prasad R, Li Calzi S, Boulton ME, Raizada MK, et al. SARS-CoV-2 Infections and ACE2: Clinical Outcomes Linked With Increased Morbidity and Mortality in Individuals With Diabetes. Diabetes (2020) 69(9):1875-86. doi: 10.2337/dbi20-0019

8. Hollstein T, Schulte DM, Schulz J, Glück A, Ziegler ME. Autoantibodynegative insulin-dependent diabetes mellitus after SARS-CoV-2 infection: a case report. Nat Met (2020). doi: 10.1038/s42255-020-00281-8

9. Marchand L, Pecquet M, Luyton C. Type 1 diabetes onset triggered by COVID-19. Acta Diabetol (2020). doi: 10.21203/rs.3.rs-38116/v1

10. Yang L, Han Y, Nilsson-Payant BE, Gupta V, Wang P, Duan X, et al. A Human Pluripotent Stem Cell-based Platform to Study SARS-CoV-2 Tropism and Model Virus Infection in Human Cells and Organoids. Cell Stem Cell (2020) 27(1):125-36 e7. doi: 10.1016/j.stem.2020.06.015

11. Fignani I, Dotta F. SARS-CoV-2 receptor Angiotensin I-Converting Enzyme type 2 is expressed in human pancreatic islet $\beta$-cells and is upregulated by inflammatory stress. (2020). doi: 10.1101/2020.07.23. 208041

12. Taneera J, El-Huneidi W, Hamad M, Mohammed AK, Elaraby E, Hachim MY. Expression Profile of SARS-CoV-2 Host Receptors in Human Pancreatic Islets Revealed Upregulation of ACE2 in Diabetic Donors. Biology (Basel) (2020) 9(8):215. doi: 10.3390/biology9080215

13. Liu F, Long X, Zhang B, Zhang W, Chen X, Zhang Z. ACE2 Expression in Pancreas May Cause Pancreatic Damage After SARS-CoV-2 Infection. Clin Gastroenterol Hepatol (2020) 18(9):2128-30. doi: 10.1016/j.cgh.2020.04.040

14. Kusmartseva I, Atkinson MA. ACE2 and SARS-CoV-2 Expression in the Normal and COVID-19 Pancreas. (2020). doi: 10.1101/2020.08.31.270736

15. Koate KC, Powers AC. SARS-CoV-2 Cell Entry Factors ACE2 and TMPRSS2 are Expressed in the Pancreas but Not in Islet Endocrine Cells. (2020). doi: 10.1101/2020.08.31.275719

16. Geravandi S, Liu H, Maedler K. Enteroviruses and T1D: Is It the Virus, the Genes or Both Which Cause T1D. MDPI Microorg (2020) 8:1017. doi: 10.3390/microorganisms8071017

17. Donath MY, Dinarello CA, Mandrup-Poulsen T. Targeting innate immune mediators in type 1 and type 2 diabetes. Nat Rev Immunol (2019) 19(12):73446. doi: 10.1038/s41577-019-0213-9

18. Del Valle DM, Kim-Schulze S, Huang HH, Beckmann ND, Nirenberg S, Wang B, et al. An inflammatory cytokine signature predicts COVID-19 severity and survival. Nat Med (2020). doi: 10.1038/s41591-020-1051-9

19. Zhang XJ, Qin JJ, Cheng X, Shen L, Zhao YC, Yuan Y, et al. In-Hospital Use of Statins Is Associated with a Reduced Risk of Mortality among Individuals with COVID-19. Cell Metab (2020). doi: 10.1016/j.cmet.2020.06.015

Conflict of Interest: The authors declare that the research was conducted in the absence of any commercial or financial relationships that could be construed as a potential conflict of interest.

Copyright (c) 2020 Ardestani and Maedler. This is an open-access article distributed under the terms of the Creative Commons Attribution License (CC BY). The use, distribution or reproduction in other forums is permitted, provided the original author(s) and the copyright owner(s) are credited and that the original publication in this journal is cited, in accordance with accepted academic practice. No use, distribution or reproduction is permitted which does not comply with these terms. 